\title{
The prevalence, reasons and attitudes for the practice of informal medicine
}

\author{
Menashe Meni Amran ${ }^{1,2^{*}}$, Avital Bilitzky Kopit ${ }^{1}$, Hannan Ariel Kranc ${ }^{2}$ and Roni Peleg ${ }^{2,3}$
}

\begin{abstract}
Background: Informal medicine, entailing undocumented medical advice, has been described in diverse medical disciplines and geographical regions. We assessed the current prevalence and characteristics of informal medical consulting, the reasons physicians provide it, and their attitudes toward it.

Methods: We conducted a survey among family physicians in Israel, a country with a national health insurance system. A questionnaire was emailed to all primary care physicians in the two largest HMOs in southern Israel. Fifteen questions addressed the prevalence, practice and attitudes to informal medical consulting. Ten questions assessed demographics and professional experience.

Results: Of 143 respondents (mean age 41 years), 55\% were women. Ninety-five percent of the respondents reported requesting informal medical consulting from other physicians. Fifty-four percent reported often providing informal consulting to family and friends; and an additional 27\% reported doing so under exceptional circumstances. The main reasons given for informal consulting requests were availability and accessibility (81\% of respondents), and not financial savings. Only $17.5 \%$ stated being in favor of informal consulting for family and friends. Only 11\% expressed feeling satisfaction after providing such consultation; 49\% expressed discomfort. Sixtysix percent thought a position paper on informal consulting to family and friends is needed.
\end{abstract}

Conclusions: Our survey of primary care physicians shows very frequent informal medical consulting, despite high dissatisfaction with such, and an interest in receiving guidelines on this practice.

Keywords: Informal medicine, Informal consulting, Primary care, Family medicine

\section{Introduction}

Informal medical consultation, in contrast to formal medical consultation, is characterized by the provision of undocumented medical advice. This includes any medical consultation or treatment provided to colleagues, family members or friends. Numerous surveys and editorials have described the intervention of physicians in the health care of family members [1]. The practice has persisted over the years, despite the inherent problems and the recommendations by such publications and by medical associations against it. In addition to family members, friends and

* Correspondence: Amran_m@mac.org.il

${ }^{1}$ Maccabi Health Services, Tel Aviv, Israel

${ }^{2}$ Ben Gurion University of the Negev, Be'er Sheva, Israel

Full list of author information is available at the end of the article persons of other relations request and receive informal medical consultation. Described as "corridor", "hallway" or "curbside" consultation, such practice has been described in diverse medical disciplines $[2,3]$ and geographical regions $[1,4]$ Self-care and self-prescribing by physicians have also been widely described [5-10].

Israel has a national health insurance system, in which all the residents are insured by one of four health maintenance organizations (HMOs). Family physician visits are at no cost. Small co-payments are charged for visits with specialists, though low socioeconomic status exempts also from these costs. During 2016, nearly 83\% of Israeli households purchased complementary health insurance from their HMOs [11]. The benefits of such include

(c) The Author(s). 2021 Open Access This article is licensed under a Creative Commons Attribution 4.0 International License, which permits use, sharing, adaptation, distribution and reproduction in any medium or format, as long as you give appropriate credit to the original author(s) and the source, provide a link to the Creative Commons licence, and indicate if changes were made. The images or other third party material in this article are included in the article's Creative Commons licence, unless indicated otherwise in a credit line to the material. If material is not included in the article's Creative Commons licence and your intended use is not permitted by statutory regulation or exceeds the permitted use, you will need to obtain permission directly from the copyright holder. To view a copy of this licence, visit http://creativecommons.org/licenses/by/4.0/ The Creative Commons Public Domain Dedication waiver (http://creativecommons.org/publicdomain/zero/1.0/) applies to the data made available in this article, unless otherwise stated in a credit line to the data. 
subsidies for second opinion consultations and scheduling appointments with shorter waiting periods.

In a survey study conducted in Israel over 20 years ago, $82 \%$ of hospital physicians reported having been asked to provide "hallway medicine"; of them, 91\% agreed [12]. However, no position paper has been issued in Israel over the last two decades to guide physicians in dealing with this phenomenon. We conducted a survey among family physicians in southern Israel, to assess the current prevalence and characteristics of informal medical consulting, the reasons physicians provide it, and their attitudes toward it.

\section{Methods}

This multicenter survey study is based on questions written by the researchers. The study population is primary care physicians (family physicians, general physicians and residents) who are employed in southern Israel. In total, 595 physicians were eligible to participate, from the two largest HMOs in Israel: 356 from Clalit Healthcare Services and 239 from Maccabi Health Services.

The questionnaire was designed to access information regarding the prevalence, reasons, means of practice and attitudes to informal medicine consultation among primary care physicians. A pilot test was performed on the initial questionnaire, among 10 participants. Following their comments, the questionnaire was revised to the final version (see Additional file 1). The questionnaire comprised 15 questions on practice and attitudes; each with 2-6 possible responses. One question asked the extent that the physicians consider each of 5 factors when approached for informal advice; the responses were on a 5 -point Likert scale. Ten questions accessed information on demographics and professional experience.

The questionnaires were sent by a link to all the email addresses of the primary care physicians affiliated with Maccabi Healthcare Services and Clalit Health Services in the southern district of Israel. A request was included on the questionnaires, that physicians should not fill the questionnaire more than once. Three reminders were sent, at intervals of $3-4$ weeks.

\section{Statistical analysis}

Statistical analysis was performed using the IBM SPSS version 25. Data were reported as means and standard deviations for continuous variables, and as percentages for categorical variables. We used the Student's t-test to determine statistically significant differences in continuous variables that were normally distributed. For continuous variables that were not normally distributed or ordinal variables, we used the Mann-Whitney test or Kruskal-Wallis test, as appropriate. The Chi-square test and Fisher's test were used to compare categorical variables. All $p$-values were two-sided and statistical significance was set at $P \leq 0.05$.

The protocol was approved by the institutional review board of Maccabi Healthcare Services and the ethical committees of Maccabi and Clalit Health Services.

\section{Results \\ Study population}

The total number of respondents was 143, for a response rate of $24 \%$. Table 1 presents the self-reported demographic characteristics of the respondents. The majority of the respondents were women, $55 \%$. The mean age was 41 years. The majority of respondents work primarily in urban clinics, $62 \%$. Half of those who stated their

Table 1 Characteristics of the respondent physicians $(N=143)$

\begin{tabular}{|c|c|c|}
\hline Characteristics & $\begin{array}{l}\text { Respondent } \\
\text { physicians }\end{array}$ & Non-responders \\
\hline \multicolumn{3}{|l|}{ Gender, \% (n) } \\
\hline Men & $44.6 \%(62)$ & 4 \\
\hline Women & $55.4 \%(77)$ & \\
\hline Age, mean $\pm S D$ & $41.3 \pm 8.8$ & 14 \\
\hline Familial status, \% (n) & & 4 \\
\hline Single & $7.9 \%(11)$ & \\
\hline Married & $89.2 \%(124)$ & \\
\hline Divorced & $2.9 \%(4)$ & \\
\hline Origin, \% (n) & & 13 \\
\hline Israel & $70.8 \%(92)$ & \\
\hline Elsewhere & $29.2 \%(38)$ & \\
\hline University, \% (n) & & 15 \\
\hline Israel & $69.5 \%(89)$ & \\
\hline Elsewhere & $30.5 \%(39)$ & \\
\hline Workplace, \% (n) & & 4 \\
\hline Urban primary care clinic & $61.9 \%(86)$ & \\
\hline Rural primary care clinic & $24.5 \%(34)$ & \\
\hline Public hospital & $5.8 \%(8)$ & \\
\hline Combination & $7.9 \%(11)$ & \\
\hline Education level / Specialization \% (n) & & 6 \\
\hline $\begin{array}{l}\text { General practitioner without } \\
\text { specialization, } \%\end{array}$ & $15.3 \%(21)$ & \\
\hline Resident & $28.5 \%(39)$ & \\
\hline Specialist in family medicine & $50.4 \%(69)$ & \\
\hline Specialist in internal medicine & $5.8 \%(8)$ & \\
\hline Seniority, median (range) & $5(0.5-45)$ & 15 \\
\hline Less than 5 years, \% (n) & $41.4 \%(53)$ & \\
\hline $6-10$ years, \% (n) & $28.9 \%(37)$ & \\
\hline More than 10 years, \% (n) & $29.7 \%(38)$ & \\
\hline
\end{tabular}

"Specialists" include specialist in family medicine and specialist in internal medicine

"Non-specialists" include resident and general practitioner without specialization 
specialty were family medicine specialists; $28.5 \%$ were residents. Forty-one percent of the respondents had less than 5 years seniority; $30 \%$ had more than ten years.

Table 2 presents the responses to the questions regarding informal medical consulting.

\section{Frequency of informal medical consulting}

The vast majority, 95\%, of the respondents reported requesting informal medical consulting from other physicians (Question 1). Thirty percent provided such service at least once daily, during the preceding month (Question 2). Only 7\% reported not providing any such consulting over the last month. Fifty-four percent of the respondents reported that they often provide informal consulting to family and friends, and an additional $27 \%$ reported doing so under exceptional circumstances (Question 6). Forty-eight percent answered that they usually accept or never refused a request for informal medical consulting from family and friends (Question 10). Seventy-five percent of the respondents stated that they highly or very highly considered their confidence in the field, when approached for informal consultation (Question 11, Fig. 1). Fifty-six percent responded that they highly or very highly considered the quality of their personal relation with the individual requesting the consultation, and $53 \%$ reported considering highly or very highly the consequences of providing incorrect advice.

\section{Characteristics of informal medical consulting}

Eighty-five percent of the respondents reported providing informal consulting face-to-face. In addition, high proportions reported also providing consultation by means of phone messages such as WhatsApp Messenger, and by electronic mail. (Question 3). The vast majority, $96 \%$, reported providing informal consulting by phone (Question 14). More than half the respondents reported providing consultation in non-emergency situations, such as interpreting medical tests or providing routine examinations (Question 7). Only 5\% of responders reported avoiding medical treatment and providing only clarification, interpretation or summation of clinical information, such as the recommendation of a specialist or the interpretation of medical. Residents and general practitioners ("non-specialists") more frequently reported interpreting medical results and recommending secondary care physicians than did specialists in internal medicine and family medicine ("specialists") (Fig. 2). The main reason presumed for the requests for informal consulting were availability and accessibility, as selected by $81 \%$ of the respondents (Question 9). Fifteen percent of the respondents reported receiving some form of compensation for providing informal medical consulting to family or friends (Question 12). No differences were found between specialists and residents in the proportions that reported refusing to provide informal consulting, and no association was found between seniority and refusing to provide consulting.

\section{Attitudes to informal medical consulting}

Only $27 \%$ responded that they were in favor of formal medical consulting for family and friends (Question 4); and only $17.5 \%$ responded being in favor of informal consulting for family and friends (Question 5). Respondents who reported receiving more requests for informal consults expressed more opposition to this type of consulting $(p=0.048)$. However, those who provided more informal consultations to family and friends expressed their support of doing such $(p=0.012)$, and their feelings following these consultations were more positive $(p<$ 0.001).

Only $11 \%$ of the respondents expressed feeling satisfaction after providing informal consultation; almost half, 49\%, expressed discomfort (Question 8). Differences were observed between men and women in their feelings after providing consultation (Fig. 3). Sixty-one percent of the women compared to $39 \%$ of the men expressed feeling uncomfortable. Thirty-six percent of the men compared to $13 \%$ of the women felt indifferent $(P=0.002)$. Of those who reported providing consultation by phone (96\%), almost half (49\%) answered that such means should only be used under emergency situations or should be avoided (Question 15). The main disadvantage to informal medical consulting according to the respondents was the lack of medical documentation, as cited by $85 \%$. Lack of objectivity and the risk of unprofessionalism or negligence were also cited by the majority of respondents (Question 13).

Sixty-six percent of the respondents thought that a position paper on informal consulting to family and friends is needed (Question 16). Such position paper was more often desired among those who expressed having negative rather than positive or indifferent feelings after providing informal consulting $(P=0.002)$; and also among those who reported more than one daily request for informal consulting $(P=0.009)$.

\section{Discussion}

This survey study revealed great dissonance among primary care physicians, between their behavior and their attitudes, in regard to informal medical consulting. On one hand, an overwhelming proportion reported involvement in such consulting, including more than half who reported frequent rates. Further, almost half reported that they usually or never refuse a request from family or friends for informal consulting. On the other hand, more than half the participants in the survey stated feeling discomfort or regret after providing informal consulting. More specific analysis of the data reveals that 
Table 2 Responses to the questionnaire

Question

2. During the last month, how often did people turn to you for informal medical consulting?

A number of times a day /Once a day

2-3 times a week/Once a week or less

Not at all

3. By what means were the requests for informal medical consults directed to you (more than one response can be selected)?

Face-to-face meeting, planned in advance

Incidental meeting such as a social event

Phone messages (such as Whatsapp)

Electronical mai

Other

4. To what degree are you in favor of formal medical consulting for family members and friends?

Strongly in favor/In favor

Neutral reaction

Not in favor/Strongly opposed

5. To what degree are you in favor of informal medical consulting for family members and friends?

Strongly in favor/In favor

Neutral reaction

Not in favor/Strongly opposed

$17.7 \%$

$36.2 \%$

$46.1 \%$

(mis $=2$ )

6. Do you give informal consulting to family and friends?

Yes, often

Yes, under exceptional circumstances

I try to avoid it/Never

7. If you answered yes on the previous question, what type of consulting to you provide to family members and friends (more than one response can be selected)?

Referrals to the emergency room

Recommendation to medical specialists

Prescriptions for drugs

$69.0 \%$

Interpretation of results of medical testing (blood tests, imaging, etc)

$81.0 \%$

Routine examinations

Treatment in emergency situations

Requests for a second opinion

8. How would you describe your feeling after providing such consultation? 
Table 2 Responses to the questionnaire (Continued)

Question
Discomfort
Regret
Other
9. What do you think are the main reasons that people turn to you informally
rather than to their family physician?
Savings in treatment costs
Accessibility and availability
Lack of trust in the public healthcare system
Confidentiality

Number
12
10
142

3
116
16
4

10. Did you ever refuse a request for informal consulting from a family member or a friend?

Yes, always/Yes, most often

Sometimes

Usually not/ Never

\section{Number}

12. Did you every receive compensation (financial or other benefits) for medical treatment or from preferring informal medicine for a family member or friend?

Yes

No

13. What do you think is the disadvantage of informal medicine (more than one response can be selected)?

A lack of medical documentation $\quad 121$

Lack of the patient's full consent $\quad 30$

Lack of objectivity

The risk of unprofessionalism or negligence

There are no particular disadvantages

14. Have you provided informal medicine by telephone or by text messaging?

Yes

No

15. If you answered yes on the last question, what is your opinion regarding such?

It's legitimate/It's not ideal, but adequate in certain situations

It's problematic and best to avoid

16. No position paper exists at this time of an ethical committee regarding the provision of informal medicine to family members and friends. Do you think such position paper is needed?

Yes $\frac{\text { Percent }}{49.3 \%}$

$8.5 \%$

$7.0 \%$

$($ mis $=1)$

$2.2 \%$

$83.5 \%$

$11.5 \%$

$2.9 \%$

(mis =4)

$7.0 \%$

$45.5 \%$

$47.6 \%$

$84.6 \%$

$85.2 \%$

$21.1 \%$

$62.0 \%$

$59.9 \%$

$3.5 \%$

(mis = 1)

95.8\%

$4.2 \%$

$51.1 \%$

$22.0 \%$

$27.0 \%$

(mis $=2$ )

$33.8 \%$

(mis $=1)$ 


\section{Physicians' considerations when approached to informal consultation (\%)}

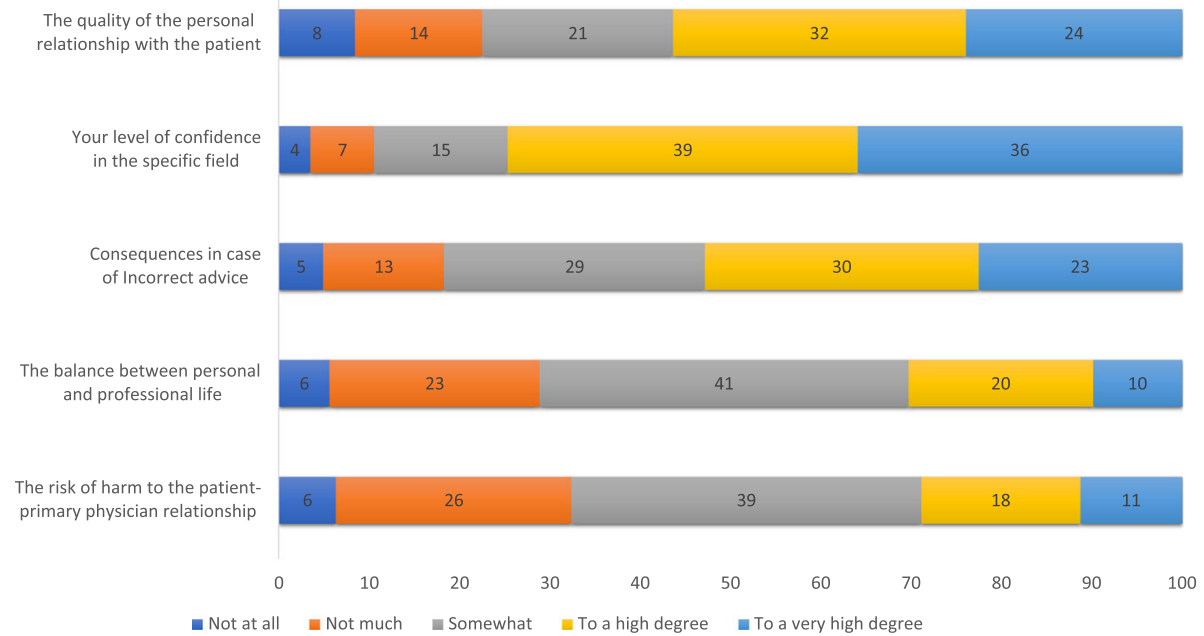

Fig. 1 Physicians' considerations when approached to informal consultation (Question 11). The numbers represent percentages as follows: the green colour represents the percentage of physicians who graded the specific consideration as 'very high level', the yellow colour represents 'high level', grey colour 'medium level', orange colour 'low level' and red colour 'not at all'. Total $N=142$

some respondents may resolve the dissonance integral to their provision of informal medical consulting. Specifically, those who reported more frequently providing informal consulting, expressed greater support of such, and their feelings following informal consultations were more positive than were those who less frequently provided such consultations.
The high proportion of physicians reporting informal consulting concurs with other studies, most of which focused on consulting to family members [1]. Nonetheless, the report by $30 \%$ of family physicians in the current survey, of providing informal consulting on a daily basis is remarkable. A particularly high prevalence of informal medicine in Israel may be related to cultural factors.

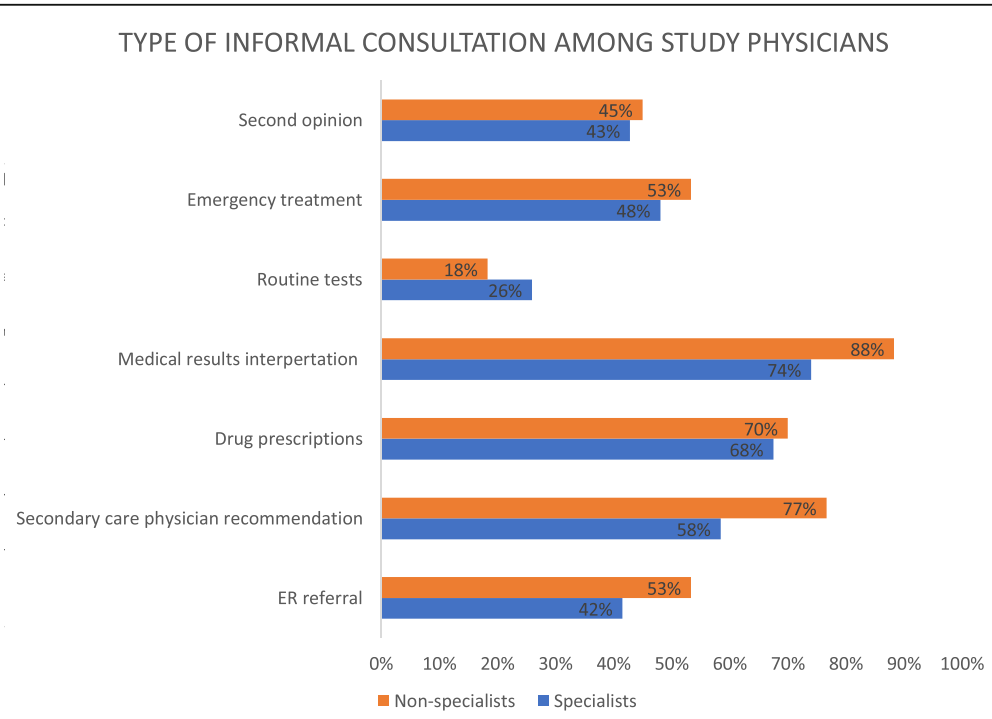

Fig. 2 Type of informal consultation among study physicians (Question 7). The orange bar represents the percentages of residents and general practitioners without specialization ("non-specialists") who reported providing each type of informal consultation. The blue bar represents the corresponding percentages among specialists (Include family medicine and internal medicine specialists). Differences were statistically significant regarding "medical results interpretation" $(P=0.037)$ and "secondary care physician recommendation" $(P=0.025)$. $P$-value was calculated using Chi-square test. ER: emergency room 


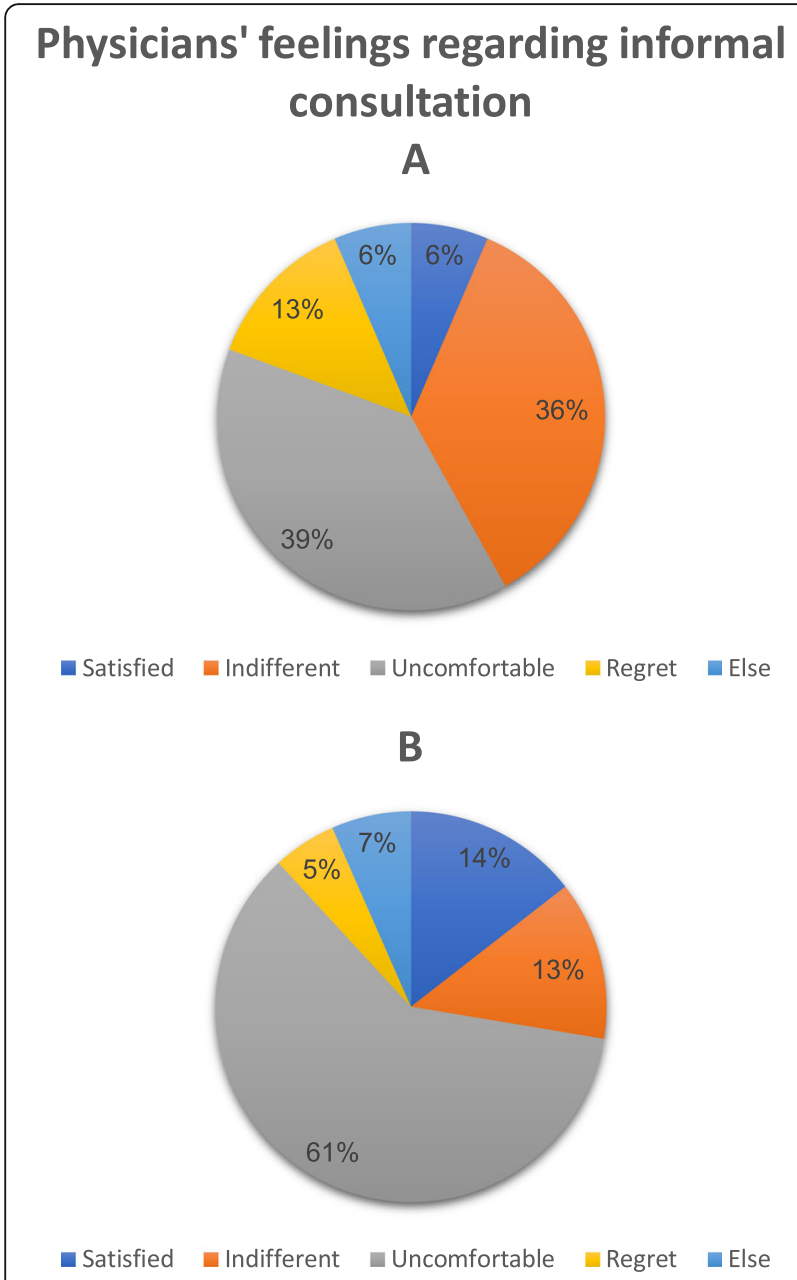

Fig. 3 Physicians' feelings regarding informal consultation (Question 8). Pie A demonstrates feelings among men while pie $B$ demonstrates feelings among women. Male physicians expressed more indifference regarding informal consultation compared to female physicians $(P=0.002)$, as calculated using the Chi-square test

Along this line, the practice in Israel of informal payments for health care has been explained in the context of a specific type of political culture, called "alternative politics" [13]. This is characterized by a "do-it-yourself" approach, which bypasses formal rules and relies on personal and reciprocal relations. The scope of this approach is broad, and may contribute to understanding the atmosphere that makes it difficult for physicians to refuse requests for informal consulting [12].

The negative attitude toward informal medical consulting expressed by the respondents of the current survey corroborates other publications [1]. Problems related to the lack in medical documentation, objectivity, and professionalism were the main disadvantages cited for informal medical consultation, concurring with the literature [1].

Only $2 \%$ of the respondents presumed that financial savings was the motivation for informal consulting. This may reflect a pervasive impact of the national health insurance system in Israel, despite the heavy reliance of the health care system on private financing [11]. In contrast, among 41 studies, financial savings was cited as a main reason for the intervention of physicians in the health care of family members [1].

In the current investigation of informal consultation, the high use reported of electronic mail and phone messages, including WhatsApp Messenger, is in agreement with the currently high use of these means of communication in formal medical consultation. WhatsApp Messenger has become a common telemedicine tool in conventional, as well as in informal medicine [14]. In a study conducted among primary care physicians in Switzerland, 82\% reported communicating with their patients by email [15]. The authors emphasized confidentiality issues as a prime disadvantage to such.

Ninety-five percent of our respondents reported requesting informal consultation from other physicians for their personal health issues. This corroborates the documentations of this phenomenon around the world, as mentioned above. Notably, a recently published cross-sectional study showed that two-thirds of hospital-based physicians in Israel do not have a regular personal physician [16].

Almost two-thirds of the respondents to our survey answered that a position paper on informal medical consulting could be beneficial. The proportion holding this attitude was particularly high among physicians who had more negative feelings after providing informal consultation and among those who reported receiving more than one daily request for informal consulting. The seventh edition of the American College of Physicians Ethics Manual, issued in 2019, [17] expanded the topic of informal medical consulting, as well as the topics on electronic communication and telemedicine ethics. Accordingly, physicians are encouraged to avoid treating themselves and family members except in emergency situations. Among the reasons cited earlier by the American Medical Association for such recommendation are difficulties in objectivity, in accessing full information and in professionalism that arise in the context of informal medical consulting [18]. Our study considered informal medicine in a broader sense than in the American College of Physicians Ethics Manual. Remarkably, 95\% of our responders reported providing informal medicine in the form of treatment and health care management, and not only clarification and interpretation of clinical information. More detailed guidelines may be beneficial to physicians, with a broader scope in regard to the nature of informal consulting, and including consulting of persons who are not family members.

No differences were found between specialists and residents in the responses to any of the items of the survey. This contrasts with the findings of a qualitative study conducted in the Netherlands, which showed more 
difficulties among junior than senior physicians in dealing with requests for informal consulting [19].

A main limitation of this survey study is selection bias, arising from the possibility that the respondents to the survey may not have been representative of the family physicians in the region examined. The questionnaire was kept short, so as to encourage respondents to fill it completely. Accordingly, very few responses were left blank. Nonetheless, the brief and structured questionnaire is limited by the information it was able to assess, compared to a more in-depth questionnaire or an interview.

\section{Conclusions}

According to a survey of family care physicians in Israel, the vast majority provide informal medical consulting to family and friends, a high proportion of them do so frequently. Discomfort and regret following such consultations were reported among many. Interest was expressed in receiving guiding principles on the matter. Due to the cultural influences inherent to informal medical consulting, more studies and specific guidelines in different geographical regions may help elucidate the problem and its consequences in various contexts. Overall, physicians seem to need more guidance and tools to help them say "No" when this is the ethical and professional response.

\section{Supplementary Information}

The online version contains supplementary material available at https:/doi. org/10.1186/s12875-020-01362-z

\section{Additional file 1}

\section{Acknowledgements}

Not applicable.

\section{Authors' contributions}

MMA was responsible for the conception of the study, for collection of the data and for preparing the first draft of the manuscript. ABK analysed the data. HAK interpreted the data and revised the manuscript. RP was involved in the design of the work and the interpretation of the data. All the authors read and approved the final manuscript.

\section{Funding}

This study was funded by Marom, a research program for physicians and residents in Maccabi Healthcare Services, and by a grant from the Israeli Association of Family Physicians.

\section{Availability of data and materials}

The datasets used and/or analysed during the current study are available from the corresponding author on reasonable request.

\section{Ethics approval and consent to participate}

The protocol was approved by the institutional review board of Maccabi Healthcare Services and the ethical committees of Maccabi and Clalit Health Services.

No patients were involved in this research. Informed consent was waived by the ethics committees of Clalit Health Services and Maccabi Health Services, because filling the questionnaires by the physicians was considered an indication of their agreement to participate.

We confirm that all the methods were performed in accordance with the relevant guidelines and regulations (Declaration of Helsinki).
Consent for publication

Not applicable.

\section{Competing interests}

The authors declare that they have no competing interests.

\section{Author details}

${ }^{1}$ Maccabi Health Services, Tel Aviv, Israel. ${ }^{2}$ Ben Gurion University of the Negev, Be'er Sheva, Israel. ${ }^{3}$ Clalit Health Services, Tel Aviv, Israel.

Received: 19 October 2020 Accepted: 10 December 2020 Published online: 03 February 2021

\section{References}

1. Scarff JR, Lippmann S. When physicians intervene in their relatives' health care. HEC Forum. 2012;24:127-37.

2. Ashique KT. Curbside (corridor to the E-corridor) consultations and the dermatologists. Indian Dermatol Online J. 2017;8:211-4.

3. Lowe S. The corridor consult. Obstet Med. 2017;10:155-6.

4. Bird S. The pitfalls of prescribing for family and friends. Aust Prescr. 2016;39:11-3.

5. Chambers R, Belcher J. Self-reported care over the past 10 years: a survey of general practitioners. Br J Gen Pract. 1992;42:153-6.

6. Wachtel TJ, Wilcox VL, Moulton AW, et al. Physicians' utilization of health care. J Gen Intern Med. 1995;10:261-5.

7. Christie JD, Rosen IM, Bellini LM, et al. Prescription drug use and selfprescription among resident physicians. JAMA. 1998;280:1253-5.

8. Hem E, Stokke G, Tyssen R, et al. Self-prescribing among young Norwegian doctors: a nine-year follow-up study of a nationwide sample. BMC Med. 2005;3:16.

9. Roberts LW, Kim JP. Informal health care practices of residents: "curbside" consultation and self-diagnosis and treatment. Acad Psychiatry. 2015:39:22-30.

10. Fekadu G, Dugassa D, Negera GZ, et al. Self-medication practices and associated factors among health-care professionals in selected hospitals of Western Ethiopia. Patient Prefer Adherence. 2020;14:353-61.

11. Achdut L. Private expenditures on healthcare: determinants, patterns and progressivity aspects. Isr J Health Policy Res. 2019;8:87.

12. Peleg A, Peleg R, Porath A, et al. Hallway medicine: prevalence, characteristics and attitudes of hospital physicians. Isr Med Assoc J. 1999;1:241-4

13. Cohen N. Informal payments for health care--the phenomenon and its context. Health Econ Policy Law. 2012;7:285-308.

14. Giordano V, Koch H, Godoy-Santos A, et al. WhatsApp messenger as an adjunctive tool for telemedicine: an overview. Interact J Med Res. 2017;6(2):e11

15. Dash J, Haller DM, Sommer J, et al. Use of email, cell phone and text message between patients and primary-care physicians: cross-sectional study in a French-speaking part of Switzerland. BMC Health Serv Res. 2016; 16:549.

16. Wilf Miron R, Malatskey L, Rosen LJ. Health-related behaviours and perceptions among physicians: results from a cross-sectional study in Israel. BMJ Open. 2019;9:e031353.

17. Sulmasy LS, Bledsoe TA. ACP Ethics, Professionalism and Human Rights Committee. American College of Physicians Ethics Manual: Seventh Edition. Ann Intern Med. 2019;170(2_Suppl):S1-32.

18. Code of Medical Ethics of the American Medical Association. Chicago: American Medical Association Press, 1847.22. The AMA Code of Medical Ethics' opinion on physicians treating family members. Virtual Mentor. 2012; 14:396-7.

19. Giroldi $E$, Freeth $R$, Hanssen $M$, et al. Family physicians managing medical requests from family and friends. Ann Fam Med. 2018;16:45-51.

\section{Publisher's Note}

Springer Nature remains neutral with regard to jurisdictional claims in published maps and institutional affiliations. 